Supporting information for

\title{
A Nanopillar Arrayed Triboelectric Nanogenerator as a Self-Powered Sensitive Sensor for a Sleep Monitoring System
}

Weixing Song, ${ }^{\dagger}$ Baoheng Gan,,$^{\dagger, \ddagger}$ Tao Jiang, ${ }^{\dagger}$ Yue Zhang, ${ }^{\dagger}$ Aifang Yu, Hongtao Yuan, ${ }^{\dagger}$ Ning Chen, ${ }^{\ddagger}$ Chunwen Sun, ${ }^{* \dagger}$ Zhong Lin Wang ${ }^{*},{ }^{\dagger} \S$

${ }^{\dagger}$ Beijing Institute of Nanoenergy and Nanosystems, Chinese Academy of Sciences; National Center for Nanoscience and Technology (NCNST), Beijing 100083, China

${ }^{\ddagger}$ School of materials science and Engineering, University of Science and Technology Beijing, Beijing 100083, China

${ }^{\S}$ School of Materials Science and Engineering, Georgia Institute of Technology, Atlanta, Georgia 30332, USA

\section{Corresponding Authors}

*E-mail: sunchunwen@binn.cas.cn.

*E-mail: zlwang@gatech.edu. 


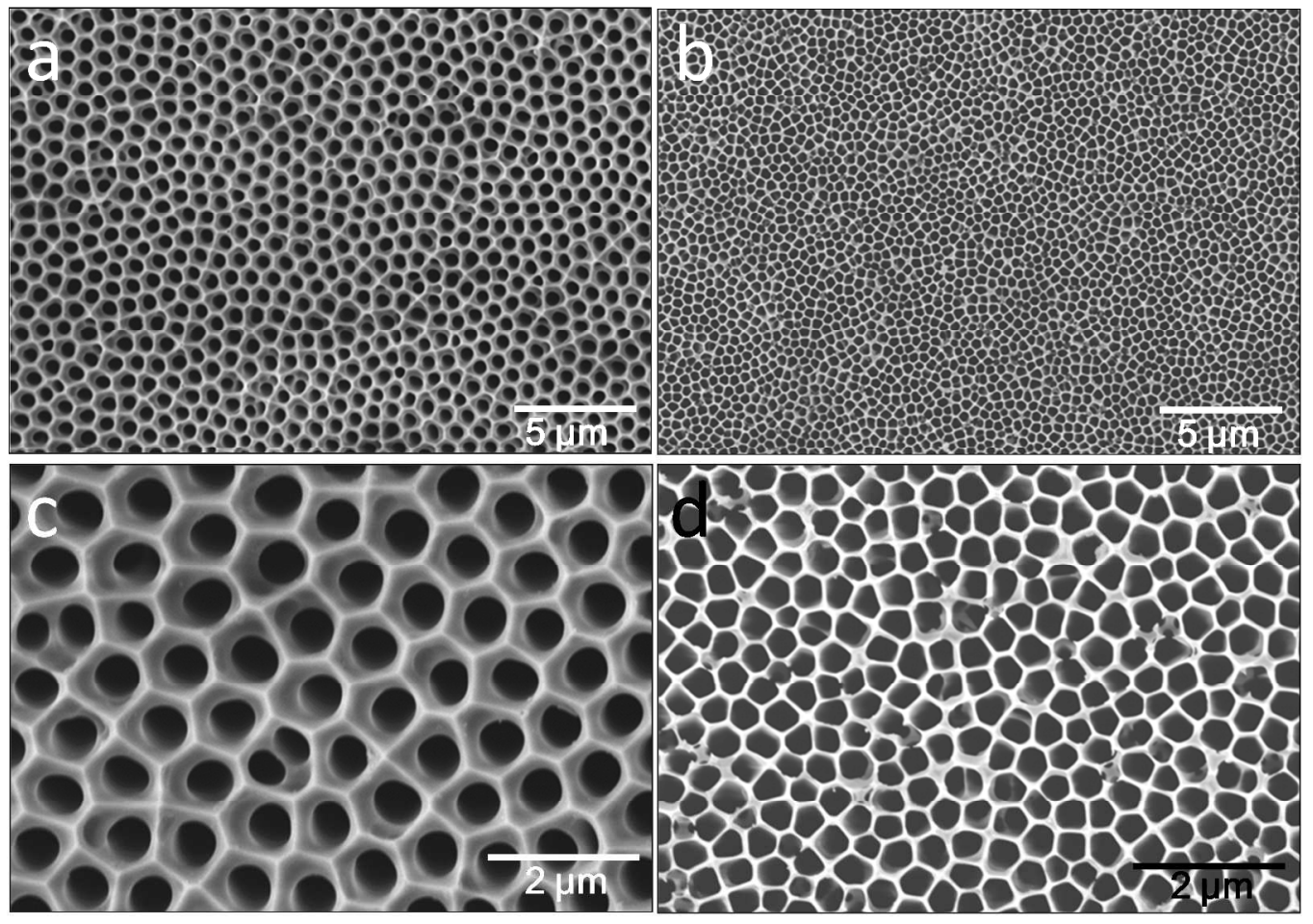

Figure S1. SEM images of AAO templates. (a) $700 \mathrm{~nm}$ diameter. (b) $400 \mathrm{~nm}$ diameter. (c) and (d) are the magnified images of (a) and (b), respectively.

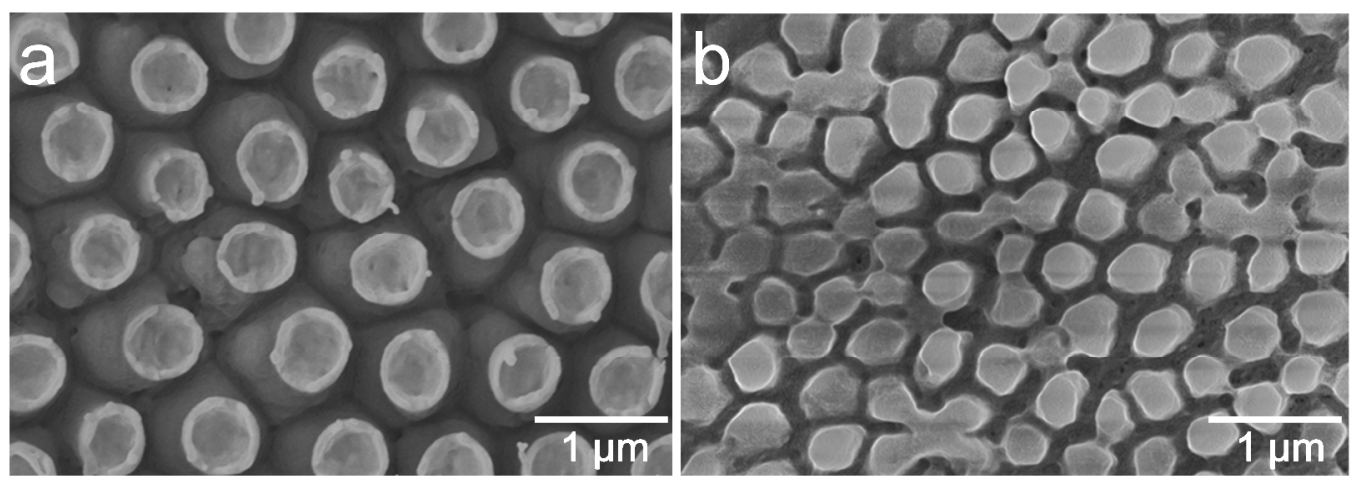

Figure S2. SEM image of (a) $350 \mathrm{~nm}$ and (b) $600 \mathrm{~nm}$ nanopillar arrays.

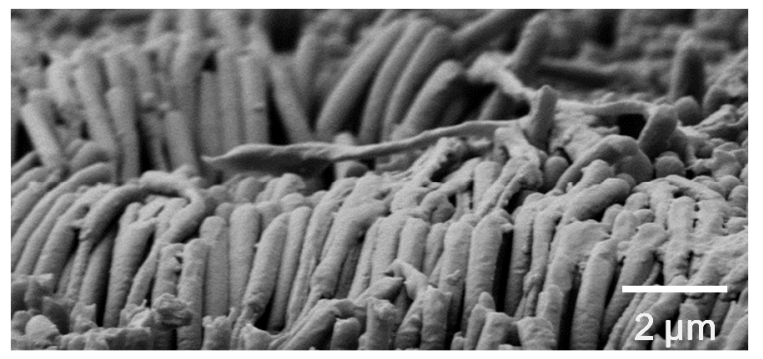

Figure S3. SEM image of nanopillar array over $3 \mu \mathrm{m}$ length. 


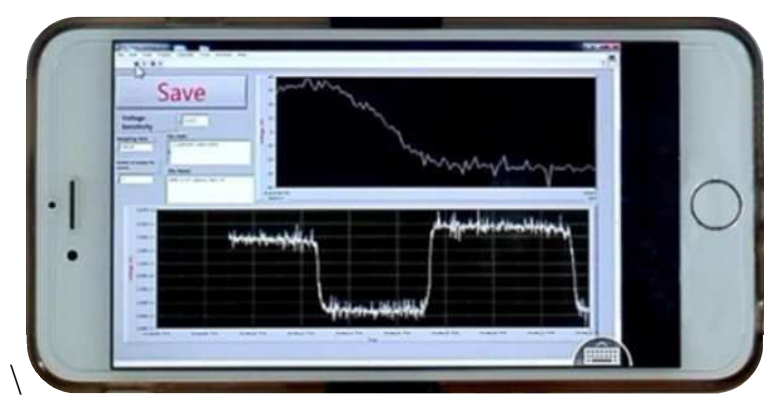

Figure S4. Photograph of the remote control and electric signals recorded by a cell phone.

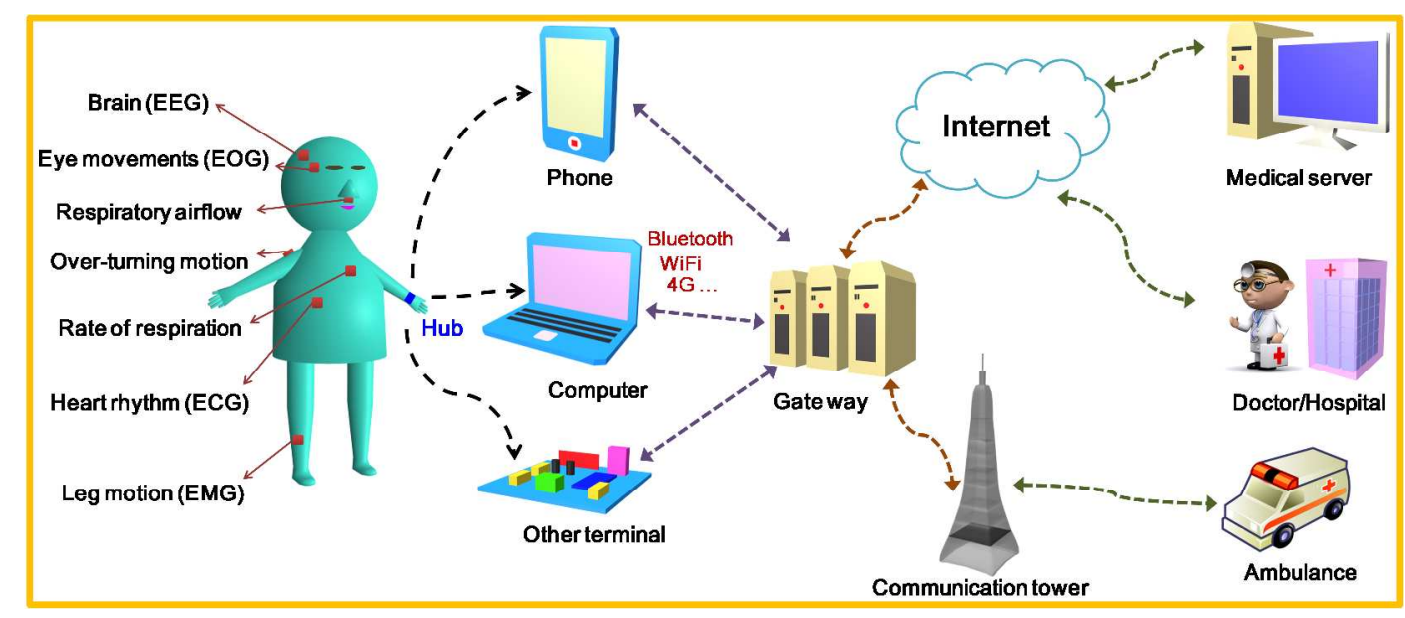

Figure S5. Schematic illustration of self-powered triboelectric sensors for wearable sleep monitoring system.

Video S1. Triggering and lighting the pocket card light powered by a TENG with a home-made cantilever spring leaf.

Video S2. Monitoring the over-turning body movement of a person during sleeping.

Video S3. Monitoring the over-turning leg movement of a person during sleeping. 